\title{
Selective-pivot sampling of radial distribution functions in asymmetric liquid mixtures
}

\author{
J. G. Malherbe \\ Physique des Liquides et Milieux Complexes, Faculté des Sciences et de Technologie, \\ Université Paris XII, 61 av. du Général de Gaulle, 94010 Créteil Cedex, France \\ Werner Krauth \\ CNRS-Laboratoire de Physique Statistique, Ecole Normale Supérieure, \\ 24 rue Lhomond, 75231 Paris Cedex 05, France
}

(Dated: 29 May 2007)

\begin{abstract}
We present a Monte Carlo algorithm for selectively sampling radial distribution functions and effective interaction potentials in asymmetric liquid mixtures. We demonstrate its efficiency for hard-sphere mixtures, and for model systems with more general interactions, and compare our simulations with several analytical approximations. For interaction potentials containing a hardsphere contribution, the algorithm yields the contact value of the radial distribution function.
\end{abstract}

\section{INTRODUCTION}

Liquid mixtures have been studied in the past decades with objectives ranging from understanding basic theoretical properties to answering questions of technological relevance. The simplest model of liquid mixtures, binary hard spheres, has been an important test bed for experimental, analytical and numerical techniques.

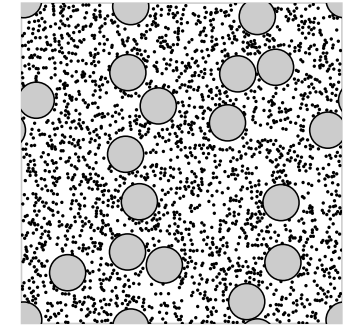

binary hard-sphere mixture

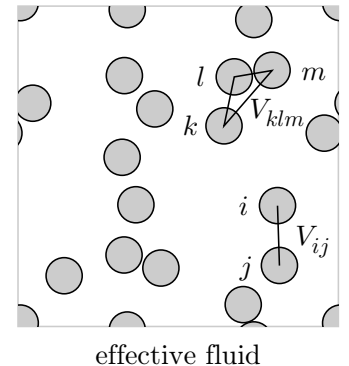

effective fluid

FIG. 1: Asymmetric hard-sphere mixture (left), and its effective fluid of solutes (pair interaction $V_{i j}$ and triplet interaction $V_{k l m}$ shown, right).

In asymmetric binary mixtures consisting of few large particles (solutes) and many small particles (solvent) (see Fig. (1), one may in principle integrate out all the degrees of freedom of the solvent, and arrive at an equivalent effective fluid of only the large particles, with complicated effective interactions involving pairs, triplets, and, more generally, $n$-tuples of solutes 11. Usually, all contributions beyond the pair interaction are neglected (see, for example, 2]), even though they are quite important [3, 4, 5]. Moreover, integral-equation theories or density functional theories [6], among others, allow to study the mixtures directly. For asymmetric hard-sphere mixtures, various approaches based on thermodynamic criteria yield empirical equations of state (see for example [7]). However, many theories may fail in extended regions of parameter space because of the different sizes of solutes and solvent (for problems with the integral-equation theories, see [8]).

Numerical simulation is a crucial tool to validate the above methods. However it also meets difficulties for asymmetric mixtures. On the one hand, the displacement of each solute is highly constrained by the surrounding solvent (see [9]). Pivot-cluster Monte Carlo algorithms [10] have partially overcome this problem for hard-sphere models [1]] and for interacting mixtures [12, 13], as long as the overall density of the system is not too high [14]. On the other hand, the difference in size of solutes and of solvent particles generates a sampling problem for observables such as the pair-distribution functions, which vary strongly on the very small scale of the solvent. To describe these observables, one needs many data (generally contained in a histogram with a fine grid). On close approach of two solutes in asymmetric mixtures, the pair correlation functions rise steeply. Therefore, the contact value of the pair-correlation function cannot be obtained precisely by extrapolation [15].

In this paper, we introduce a selective-pivot sampling algorithm, which allows us to compute the ratio of the radial distribution functions $g(R) / g\left(R^{\prime}\right)$ for two arbitrary inter-particle distances $R$ and $R^{\prime}$. For hard-sphere mixtures, we compute the pair-correlation function of the solutes and especially its contact value. We also determine the potential of the mean force. We furthermore discuss the extension of the method to systems with arbitrary pair interactions. 


\section{SELECTIVE-PIVOT SAMPLING}
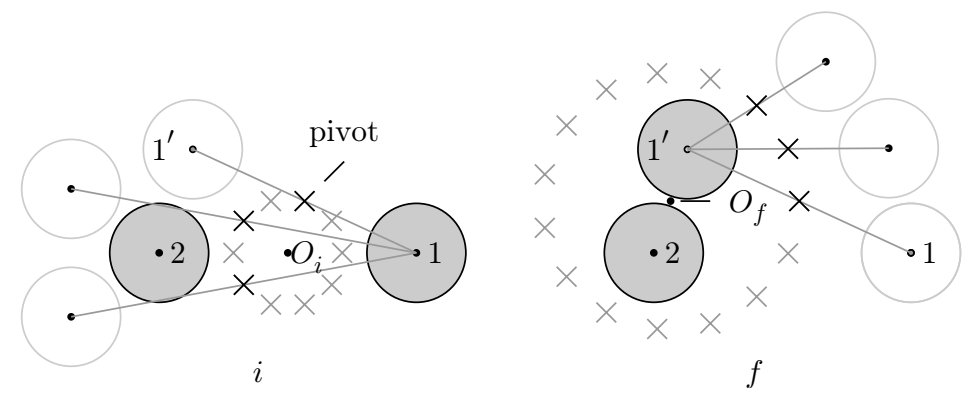

FIG. 2: Selective-pivot move $1 \rightarrow 1^{\prime}$ (configuration $i$, left) and return move $1^{\prime} \rightarrow 1$ ( $f$, right). In $i$, the distance $R_{12}=R_{i}$, and $R_{1^{\prime} 2}=R_{f}$. All pivots resulting in a new distance $R_{f}$ lie on a sphere $S_{i}$ with center $O_{i}$ and radius $R_{f} / 2($ crosses $)$.

For simplicity, we first discuss selective-pivot sampling for a system of an isolated pair of three-dimensional hard spheres. To sample the ratio of the pair correlation function for two arbitrarily distances, $R_{i}$ and $R_{f}$, we consider a configuration $i$, with a distance $R_{i}$ between the two particles, and all moves of one particle $\left(1 \rightarrow 1^{\prime}\right)$ such that the new distance is $R_{f}$ (see Fig. 2). Any such move can be interpreted as a reflection around a pivot located on a sphere $S_{i}$ with radius $R_{f} / 2$ and center $O_{i}$, half-way between the two particles. We choose the pivot randomly on the sphere $S_{i}$ (using Gaussian random numbers, see [9]). The a priori probabilities for proposing the move $i \rightarrow f$, and the return move $f \rightarrow i$ are, respectively,

$$
\mathcal{A}(i \rightarrow f)=\frac{1}{S_{i}} \propto \frac{1}{R_{f}^{2}}, \quad \mathcal{A}(f \rightarrow i)=\frac{1}{S_{f}} \propto \frac{1}{R_{i}^{2}} .
$$

The detailed-balance condition connects the stationary probabilities $\pi(i)$ and $\pi(f)$ with the a priori probabilities:

$$
\pi(i) \mathcal{A}(i \rightarrow f)=\pi(f) \mathcal{A}(f \rightarrow i) .
$$

It follows that the probabilities of the two configurations are biased by a geometric factor with respect to the constant hard-sphere probabilities,

$$
\frac{\pi(i)}{\pi(f)}=\frac{S_{f}}{S_{i}}=\frac{R_{f}^{2}}{R_{i}^{2}} .
$$

For spherically symmetric systems, the pair correlation function depends solely on the distance between particles and reduces to the radial distribution function $g(R)$, which is linked with the probability $\pi(R)$ to observe a particle in an infinitesimal spherical shell of radius $R$, the other one being at its center, by

$$
\pi(R) \propto R^{2} g(R) \mathrm{d} R .
$$

The biasing factor in eq. (1) cancels the phase-space factor of the radial distribution function, and the ratio of the probabilities $\pi(i)$ and $\pi(f)$ equals the ratio of the radial distribution functions:

$$
\frac{\pi(i)}{\pi(f)}=\frac{g_{i}}{g_{f}} .
$$

The above algorithm and the relation of eq. (2) between the probabilities of observing the distances $R_{i}$ and $R_{f}$ remain valid for two or more solute particles in a box with periodic boundary conditions, in the presence of other components (solvent). It suffices to permanently tag two solutes. One Monte Carlo move involves a single pivot, but may transform many particles (see [9]), including both tagged ones, so that the distance between them may not change.

\section{APPLICATIONS}

To validate the selective-pivot sampling algorithm, and to explore possible applications, we compute the distribution function of the solutes for asymmetric binary mixtures, and compare it with simulation data obtained with standard cluster simulation methods, as well as with analytic approximations. In this system, we also compute the contact value of the distribution function. This can be done directly, without extrapolation. Furthermore, we use the selective-pivot sampling algorithm to compute the potential of the mean force, that is, the distribution function for two solutes in a large bath of solvent, approaching the regime of infinite dilution. 


\section{A. Radial distribution function of solutes}

We consider an asymmetric binary mixture of spheres with diameters $D_{\mathrm{s}}$ (small particles - solvent) and $D_{\mathrm{l}}$ (large particles-solute), and size ratio $R=D_{\mathrm{l}} / D_{\mathrm{s}}=10$, at packing fractions $\eta_{\mathrm{s}}=0.126$ and $\eta_{\mathrm{l}}=0.121$, where the cluster algorithm performs well (the packing fraction of the solvent, $\eta_{\mathrm{s}}$, is linked to the number of solvents $N_{\mathrm{s}}$ in the simulation box of volume $V$ by $\eta_{\mathrm{s}}=\frac{\pi}{6}\left(N_{\mathrm{s}} / V\right) D_{\mathrm{s}}^{3}$, etc. $)$.

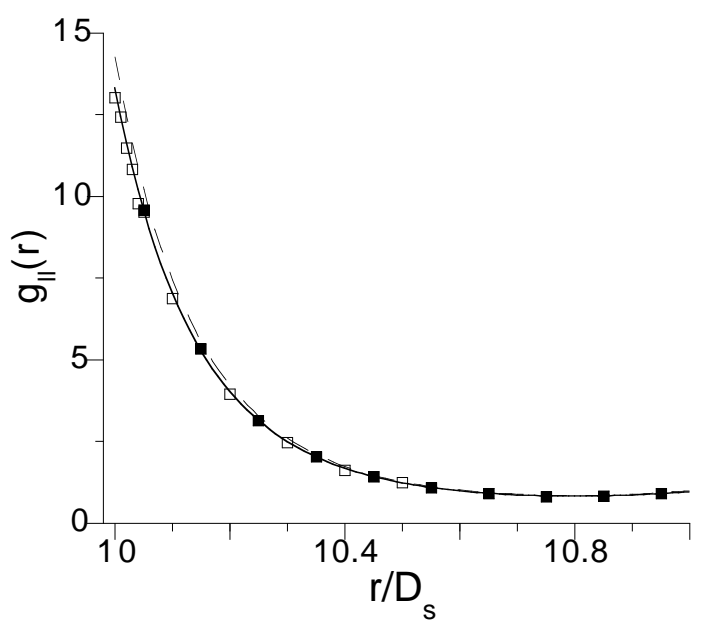

FIG. 3: Radial distribution function of the solutes for hard spheres with $R=10, \eta_{\mathrm{s}}=0.126$ and $\eta_{1}=0.121$. Symbols: Cluster algorithm (filled squares: standard sampling method; empty squares: selective-pivot sampling); full line: density functional theory; dashed line: RHNC-MSA [8]

In Fig. 3, we compare the radial distribution function of the large spheres $g_{11}(r)$ obtained from selective-pivot sampling and from a standard cluster algorithm and find very good agreement. The contact region and, especially, the contact value $g_{11}\left(r=D_{1}\right)$ become accessible only with our sampling scheme. With standard grid sampling, they can be obtained only by extrapolation because the probability of observing two solutes exactly at contact is zero. Our sampling approach gives sensibly more accurate contact values than the standard method (see [16]). In Fig. 3. $g_{11}\left(D_{1}\right)=13.03 \pm 0.26$. As a common use of simulation data, we also compare the pair-correlation function with density functional theory, which agrees very well [17, 18], and with the Ornstein-Zernike integral equation with the RHNC-MSA closure, which overshoots in the contact region [8].

\section{B. Potential of the mean force}

As another application of selective-pivot sampling, we consider the potential of the mean force, the solute paircorrelation function in the limit of infinite dilution of the solute particles:

$$
\lim _{\eta_{1} \rightarrow 0} g_{11}\left(r, \eta^{\mathrm{b}}\right)=\exp [-\beta U(r)]
$$

where $U(r)=u_{11}(r)+\Phi_{\text {eff }}(r)$ is the total pair interaction between the solutes, $u_{11}(r)$ the direct interaction, $\Phi_{\text {eff }}(r)$ the pair potential of the mean force and $\eta_{b}$ the bulk solvent packing fraction. The selective-pivot sampling algorithm allows us therefore to compute the potential of the mean force of the effective fluid obtained by integrating out the solvent particles of the binary mixture. One simply puts the two tagged solutes into a bath of solvent and determines the potential differences for several pairs $R_{i}, R_{f}$. The size of the bath plays almost no role in this simulation because the two large particles are kept at two fixed distances, and cannot escape to infinite separation. The simulation can thus take place in a large box, approaching the infinite dilution of the two solute particles.

To illustrate this point, we computed the potential of the mean force between two hard-sphere solutes in a bath of hard-sphere solvent with size ration $R=5$ and bulk packing fractions $\eta_{\mathrm{b}}=0.1$ and $\eta_{\mathrm{b}}=0.2$. We considered here $N_{\mathrm{s}}=5280$ and $N_{\mathrm{s}}=10560$ respectively in a cubic box of $L=30 D_{\mathrm{s}}$. At a difference of other simulation methods for computing the effective interaction, this method needs no extrapolation [19] and has no adjustable parameter [20]. The Monte Carlo data agree very well with the potential of the mean force obtained from the Ornstein-Zernike equation with the Rosenfeld fundamental measure closure [21, 22] (see Fig. 44). 


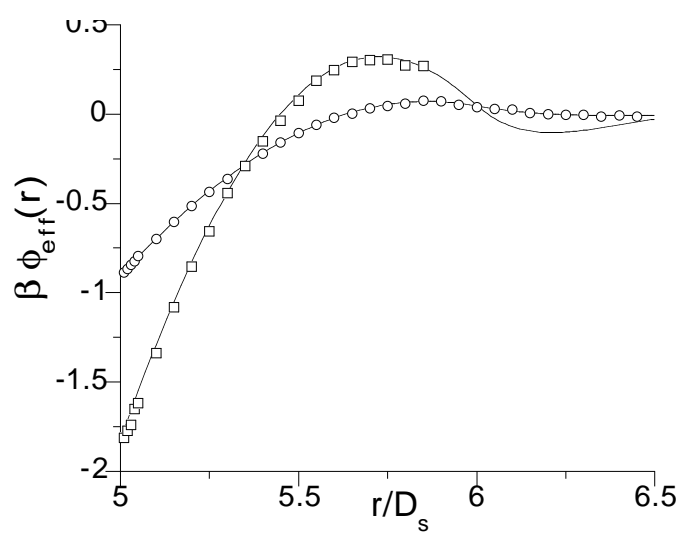

FIG. 4: Potential of the mean force for hard-sphere mixture with $R=5$; symbols: Selective-pivot sampling; lines: RHNC/fundamental measure functional; circles: bulk packing fraction $\eta_{\mathrm{b}}=0.1 ;$ squares: $\eta_{\mathrm{b}}=0.2$.

\section{SELECTIVE-PIVOT SAMPLING FOR MODELS WITH GENERAL PAIR INTERACTIONS}

Our sampling method can be directly generalized to more general interactions, which can also be handled by the pivot-cluster algorithm (see [12, 13]). As an illustration we study the effect of solvation forces in a binary mixture of colloids with size ratio $R=5$, and a Yukawa tail for unlike pairs of colloids added to the hard-sphere interaction:

$$
u_{\mathrm{ls}}(r)= \begin{cases}\infty & r<D_{\mathrm{ls}} \\ -\epsilon_{\mathrm{ls}} \exp \left\{-z_{\mathrm{ls}}\left(r-D_{\mathrm{ls}}\right)\right\} / r & r \geq D_{\mathrm{ls}}\end{cases}
$$

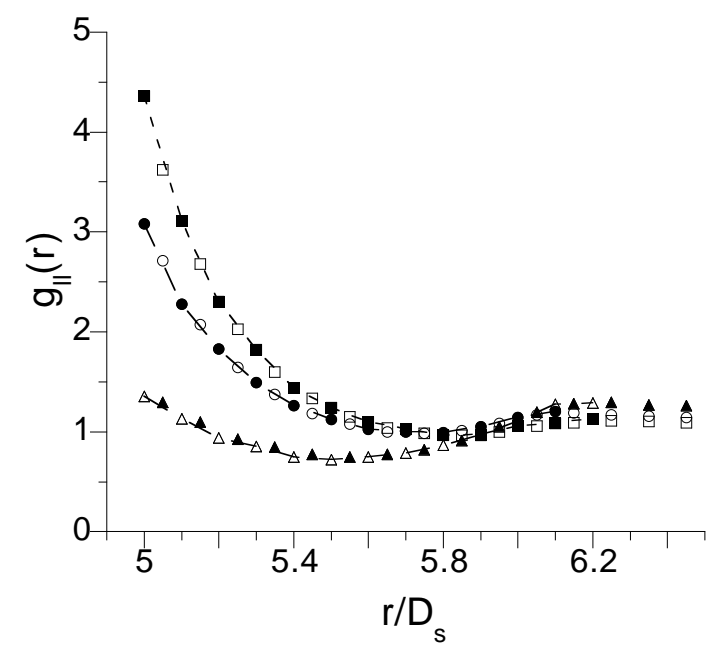

FIG. 5: Pair distribution function of solute particles for $R=5, \eta_{\mathrm{s}}=0.11$ and $\eta_{1}=0.12$. Empty symbols: standard sampling method; filled symbols: selective-pivot sampling; squares: hard-sphere; circles: Yukawa interaction with $z_{\mathrm{ls}}^{*}=10$; triangles: with $z_{\mathrm{ls}}^{*}=2.5$.

We used a value of $0.5 k_{\mathrm{B}} T$ for the depth of the attractive well, which correspond to $\epsilon_{\mathrm{ls}}^{*}=\epsilon_{\mathrm{ls}} D_{\mathrm{s}} /\left(k_{\mathrm{B}} T\right)=3 / 2$, and

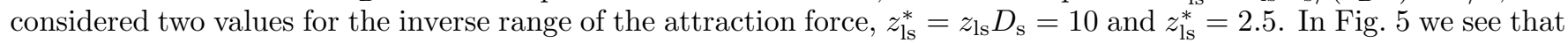
data obtained from selective-pivot sampling and from standard cluster algorithm agree very well. The advantages and the applications mentioned in the previous section carry over. Fig. [5 shows that the contact value of the radial distribution function decreases when the range of the attraction between small and large particles increases, because the solvation forces create a thin shell of small colloids which counterbalances the depletion between the large ones. 


\section{CONCLUSION}

In this paper, we have presented a selective sampling cluster algorithm which allows to obtain the radial distribution function, including the contact value, and also yields the pair potential of the mean force between two solutes due to the presence of the solvent. The algorithm solves a sampling problem for observables with very rich structure on a small scale, but does not overcome the remaining limitation of cluster algorithms for liquid simulations, namely the restriction to moderate densities. It will be interesting to see whether this restriction can also be overcome in the future.

[1] T. L. Hill, Statistical Mechanics (Dover Publications, New York) (1987).

[2] M. Dijkstra, R. van Roij, and R. Evans, Phys. Rev. E 59, 5744 (1999).

[3] R. Tehver, A. Maritan, J. Koplik, and R. Banavar, Phys. Rev. E 591339 (1999).

[4] J. G. Malherbe and S. Amokrane, Molec. Phys. 99, 355 (2001).

[5] S. Amokrane, A. Ayadim, and J. G. Malherbe, J. Phys.: Condens. Matter 15, S3443 (2003).

[6] R. Evans, Fundamentals of inhomogeneous fluids, Ch.3, ed D. Henderson (Marcel Dekker New York), 85 (1992).

[7] D. Henderson and K. Y. Chan, Molec. Phys. 98, 1005 (2000); C. Regnaut, A. Dyan, and S. Amokrane, Molec. Phys. 99, 2055 (2001); D. Viduna and W. R. Smith, Molec. Phys. 100, 2903 (2002); A Santos, S. B. Yuste, and M. López de Haro, J. Chem. Phys. 117, 5785 (2002); C. Barrio and J. R. Solana, J. Chem. Phys. 119, 3826 (2003).

[8] S. Amokrane, A. Ayadim, and J. G. Malherbe, J. Chem. Phys. 123, 174508 (2005).

[9] W. Krauth, Statistical Mechanics: Algorithms and Computations (Oxford University Press, Oxford, UK) (2006).

[10] C. Dress and W. Krauth, J. Phys. A: Math. Gen. 28, L597 (1995).

[11] A. Buhot and W. Krauth, Phys. Rev. Lett. 80, 3787 (1998).

[12] J.G. Malherbe and S. Amokrane, Molec. Phys. 97, 677 (1999).

[13] J. Liu and E. Luijten, Phys. Rev. Lett. 92, 035504 (2004).

[14] J. Liu and E. Luijten, Phys. Rev. E 71, 066701 (2005).

[15] M. P. Allen and D. J. Tildesley, Computer simulation of liquids (Oxford Science Publication, UK) (1987).

[16] A. Vrabecz and G. Tóth, Molec. Phys. 104, 1843 (2006).

[17] Y. Yu and J. Wu, J. Chem. Phys. 117, 10156 (2002).

[18] R. Roth, R. Evans, A. Lang, and G. Kahl, J. Phys.: Condens. Matter 4, 12063 (2002).

19] R. Dickman, P. Attard, and V. J. Simonian, J. Chem. Phys. 107, 205 (1997).

[20] W. Li and H. R. Ma, Phys. Rev. E 66, 061407 (2002).

[21] Y. Rosenfeld, J. Chem. Phys. 98, 8126 (1993).

[22] S. Amokrane and J. G. Malherbe, J. Phys.: Condens. Matter 13, 7199 (2001); erratum ibid 14, 3845 (2002). 